\title{
A Search for Tools to Support Decision-Making for PIVC Use
}

\author{
Mary Alexander, MA, RN, CRNI@, CAE, FAAN*
}

Infusion Nurses Society and Infusion Nurses Certification Corporation, Norwood, Massachusetts.

Peripheral intravenous catheters (PIVCs) are the most frequently used vascular access devices (VADs) in all patient populations and practice settings. Because of its invasive nature and the fact that PIVCs are placed and medications are administered directly into the bloodstream, vascular access is risky. There are multiple factors to consider when placing a PIVC, the least of which is determining the most appropriate device for the patient based on the prescribed therapy.

VAD planning and assessment needs to occur at the first patient encounter so that the most appropriate device is selected and it aligns with the duration of the treatment, minimizes the number of unnecessary VADs placed, and preserves veins for any future needs. The level of the clinician's expertise, coupled with challenging environments of care, add to the complexity of what most perceive to be a "simple" procedure-placing a PIVC. For these reasons, it's imperative that clinicians are competent in the use and placement of VADs to ensure safe patient care.

Carr and colleagues ${ }^{1}$ performed a notable scoping review to determine the existence of tools, clinical prediction rules, and algorithms (TRAs) that would support decision-making for the use of PIVCs and promote first-time insertion success (FTIS). They refined their search strategy to studies that described the use or development of any TRA regarding PIVC insertion in hospitalized adult patients.

The team identified 36 references for screening and based on their inclusion and exclusion criteria, were left with 13 studies in the final review. Inclusion criteria included TRAs for PIVC insertion in hospitalized adult patients using a traditional insertion approach, which was defined as "an assessment and/or insertion with touch and feel, therefore, without vessel locating technology such as ultrasound and/ or near infrared technology." ${ }^{1}$ Of note is that some of the exclusion criteria included pediatric studies, TRAs focused on postinsertion assessment, studies that examined VADs other than PIVCs, and studies in which vascular visualization techniques were used.

In general, the authors were unable to find reported evidence that the study recommendations were adopted in clinical practice or to what degree any TRA had on the success

\footnotetext{
*Address for correspondence and reprint requests: Mary Alexander, MA, RN, CRNI ${ }^{\circledR}$, CAE, FAAN, Infusion Nurses Society, 315 Norwood Park South, Norwood, MA 02062; Telephone: 781-440-9408; Fax: 781-440-9409; E-mail: mary.alexander@ins1.org
}

Received: July 22, 2017; Accepted: July 24, 2017

2017 Society of Hospital Medicine DOI 10.12788/jhm.2840 of a PIVC insertion. As a result, they were unable to determine what, if any, clinical value the TRAs had.

The review of the studies, however, identified 3 variables that had an impact on PIVC insertion success: patient, clinician, and product characteristics. Vein characteristics, such as the number, size, and location of veins, and patients' clinical conditions, such as diabetes, sickle cell anemia, and intravenous drug abuse, were noted as predictors of PIVC insertion success. In 7 papers, the primary focus was on patients with a history of difficult intravenous access (DIVA). The definition of DIVA varied from time to insertion of the PIVC to the number of failed attempts, ranging from 1 to 3 or more attempts.

Clinician variables, such as specialty nurse certification, years of experience, and self-reporting skill level, were associated with successful insertions, and clinicians who predicted FTIS were likely to have FTIS. Product variables included PIVC gauge size and the number of vein options and the relationship with successful first attempts.

Limitations noted by the researchers were a lack of sufficient published evidence for TRAs for PIVC insertion and standardized definitions for DIVA and expert inserters. The number of variables and the dearth of standardized terms may also influence the ability to adopt any TRAs.

While the purpose of the research was to identify TRAs that could guide clinical practice for the use of PIVCs and successful insertions, the authors make an important point that dwell time was not considered. While a TRA may lead to a successful insertion, it may not transcend the intended life of the PIVC or the duration of the therapy. Therefore, TRAs should embed steps that ensure the appropriate device is selected at the start of the patient's treatment.

The authors identified a need for undertaking and providing research in a critical area of patient care and safety. This article increases awareness of issues related to PIVCs and the impact they have on patient care. FTIS rates vary and the implications of their use are many. Patient satisfaction, no delay in treatment, vein preservation, a decreased risk of complications, and the cost of labor and products are factors to consider. Tools to improve patient outcomes related to device insertion, care, and management need to be developed and validated. The authors also note that future TRAs should integrate the use of ultrasound and vascular visualization technologies.

In a complex, challenging healthcare environment, tools and guidance that enhance practice do not only help clinicians; they have a positive impact on patient care. The need for research, so that gaps in knowledge and science can be bridged, is 
clear. Gaps must be identified, research conducted, and TRAs developed and adopted to enhance patient outcomes.

Disclosure: The author reports no conflicts of interest.

\section{Reference}

1. Carr PJ, Higgins NS, Rippey J, Cooke ML, Rickard CM. Tools, clinical prediction rules, and algorithms for the insertion of peripheral intravenous catheters in adult hospitalized patients: a systematic scoping review of literature. J Hosp Med. 2017; $12(10): 851-858$ 